\title{
The 8th Supplement of the Italian Spine Society/Scoliosis Study Group (SICV\&GIS)
}

\author{
Marco Crostelli ${ }^{1}$ Alberto Di Martino ${ }^{2,3} \cdot$ Giuseppe Costanzo $^{4}$
}

Received: 26 May 2018 / Accepted: 27 May 2018 / Published online: 6 June 2018

c) Springer-Verlag GmbH Germany, part of Springer Nature 2018

Spine surgery in Italy has greatly evolved in the last few decades in terms of surgical techniques, equipment, instrumentation systems, and ability to define and manage complex clinical pictures. In fact, any physician dealing with spinal diseases must be aware of the expected consequences of their management, to understand the course of the disease. Such an awareness will allow the surgeons to undertake the appropriate measures necessary for the best patient care and therefore to improve the outcomes. Spine surgery typically represents a field of shared competence between orthopaedic surgeons and neurosurgeons, even though the recent interest of the scientific community towards the management of spinal deformities has seen a renewed interest by the orthopaedic community towards it.

This Supplement shows current approaches to various spinal problems: degenerative spine diseases, fractures, infections and deformities. We are pleased that the Supplement explores some "cutting edge" topics such as the recent advances in three-planar spinal deformity corrections, as well as alternative methods of deformity corrections.

We also emphasize a newer vertebral body necrosis classification system and the guidelines for its management, aimed to the recognition of this poorly understood disease; hopefully the proposed classification may drive the clinicians to its recognition and suggest a standardized management.
Another paper explores the contest of pyogenic spinal infections, the epidemiological and clinical features, the prognostic factors and the long-term outcomes in a wide cohort of patients.

We also selected manuscripts on the topics of trauma that regard the role of vertebroplasty in respect to the conservative treatment, the extra-spinal associated lesions, and the performance of different instrumentations in these settings of patients.

Other manuscripts show a contemporary approach to minimally invasive spine surgery in the setting of the degenerative spine; the positive outcomes are the results of standardized approaches and careful selection of patients.

We believe that the content of this Supplement will be interesting to the surgeons dedicated to the treatment of spine diseases.

We would like to thank all the contributing authors for their interesting manuscripts. Once more, these articles outline the clinical and scientific standards of the Italian Spine Community.

\section{Compliance with ethical standards}

Conflict of interest The authors declare no conflit of interest.

Marco Crostelli

marco.crostelli@opbg.net

1 Spine Disease Unit, Ospedale Pediatrico Bambino Gesù, Via Timavo, 32, 00195 Rome, Italy

2 Department of Orthopaedics and Trauma Surgery, University Campus Bio-Medico of Rome, Rome, Italy

3 Sidney Kimmel Medical College of Thomas Jefferson University (SKMC), Philadelphia, USA

4 Orthopaedics, La Sapienza University of Rome, Rome, Italy 